\title{
Chiral Disorder in Two-Color QCD with Abelian External Fluxes
}

\author{
Romuald A. Janik ${ }^{\mathrm{a}, b}$, Maciej A. Nowak ${ }^{b, c}$, Gábor Papp $^{\mathrm{d}}$, and Ismail Zahed ${ }^{\mathrm{e}}$ \\ aService de Physique Théorique, CEA Saclay, F-91191 Gif-Sur-Yvette, France \\ ${ }^{b}$ Marian Smoluchowski Institute of Physics, Jagellonian University, 30-059 Krakow, Poland \\ ${ }^{\mathrm{c}}$ GSI, Planckstr. 1, D-64291 Darmstadt, Germany \\ ${ }^{\mathrm{d}}$ CNR Department of Physics, KSU, Kent, Ohio 44242, USA \& \\ HAS Research Group for Theoretical Physics, Eötvös University, Budapest, Hungary \\ ${ }^{\mathrm{e}}$ Department of Physics and Astronomy, SUNY, Stony Brook, New York 11794, USA
}

\begin{abstract}
We investigate the effects of several Abelian external fluxes $\phi$, on the Euclidean Dirac spectrum of light quarks in QCD with two colors. Our results provide for a novel way of assessing the pion weak decay constant directly from spectral fluctuations, and discriminating a flux-rich from a flux-poor vacuum, using QCD lattice simulations.

PACS: 11.30.Rd, 12.38.Aw, 64.60.Cn
\end{abstract}

\section{Introduction}

In two-color QCD the quarks are in the pseudoreal representation of the flavor group [1,2]. For two flavors, the spontaneous breaking of chiral symmetry is accompanied by the occurrence of five Goldstone modes: 3 pions and a baryon and an antibaryon. The five Goldstone modes decay weakly, with a mass that satisfies the Gell-MannOakes-Renner (GOR) relation [1]. The latter mass vanishes in the chiral limit.

In this talk we consider the effects of several Abelian external fluxes on the light quarks in a finite Euclidean volume with two-color QCD. We will show that these fluxes alter the quark spectral correlations near zero virtuality in a way that is sensitive to the bulk conductance of the disordered system [3], hence to the pion decay constant. In a finite volume and in current lattice simulations, this provides for a novel way to measure the pion decay constant. Our analysis in QCD will parallel recent analysis of electrons in disordered metals [4]. A more thorough discussion can be found in [5].

\section{QCD with Abelian fluxes}

In a finite Euclidean volume $V=L^{4}$ pierced by Abelian fluxes $\phi_{\mu}=\left(\phi_{1}, \phi_{2}, \phi_{3}, \phi_{4}\right)$, the light quarks in the background gluon field $A$ satisfy the following Dirac equation

$i \not \varnothing[A] q_{k}=\lambda_{k}[A, \phi] q_{k}$.

subject to the boundary condition

$q_{k}\left(x+L_{\mu}\right)=-e^{i 2 \pi \phi_{\mu}} q_{k}(x)$.

The $\phi$ 's are given in units of a flux quantum $h / e_{q}$ set to 1 for convenience. Through (2) the quark spectrum (11) depends explicitly on $\phi$.

The probability $p(t, \phi)$ for a light quark to start at $x(0)$ in $V$ and return back to the same position $x(t)$ after a proper time duration $t$, is

$p(t, \phi)=\frac{V^{2}}{N}\left\langle\left|\left\langle x(0)\left|e^{i(i \not\lceil[A]+i m)|t|}\right| x(0)\right\rangle\right|^{2}\right\rangle_{A}$.

The averaging in (3) is over all gluon configurations using the unquenched two-color QCD measure The normalization is per state, where $N$ is the total number of quark states in the fourvolume $V$. Eq. (3) may be written in terms of 
the standard Euclidean propagators for the quark field,

$$
\begin{aligned}
& p(t, \phi)=\frac{V^{2}}{N} \lim _{y \rightarrow x} \int \frac{d \lambda_{1} d \lambda_{2}}{(2 \pi)^{2}} e^{-i\left(\lambda_{1}-\lambda_{2}\right)|t|} \\
& \times\left\langle\operatorname{Tr}\left(S\left(x, y ; z_{1}, \phi\right) S^{\dagger}\left(x, y ; z_{2}, \phi\right)\right)\right\rangle_{A}
\end{aligned}
$$

generalizing to $\phi \neq 0$ the results in [3]. Here $z_{1,2}=m-i \lambda_{1,2}$, and

$S\left(x+L_{\mu}, y ; z, \phi\right)=-e^{i 2 \pi \phi_{\mu}} S(x, y ; z, \phi)$.

The correlation function in (4) relates (in general) to an analytically continued pseudoscalar correlation function, as the eigenvalues $q_{k}$ and $-\gamma_{5} q_{k}$ are pair-degenerate (chiral) [3]. For twocolor QCD there is an extra symmetry [1,2] that makes the pseudoscalar correlation function degenerate with certain diquark correlation functions in the flux-free case. Indeed, for $\phi=0$ and $\mathbf{K}=-\tau^{2} C K$ the eigenvalues $q_{k}$ and $\mathbf{K} q_{k}$ are also pair-degenerate. Here $C$ is the chargeconjugation matrix, $\tau^{2}$ the color matrix and $K$ the (right-left) complex-conjugation.

For $\phi \neq 0$, the first symmetry (chiral) is retained while the second one is upset [6]. The quarks are now required to be in the complex representation, except for the case where $\phi$ is halfinteger. The fluxes add in the cooperon channel (diquark) with net Abelian charge 2, but cancel in the diffuson (pseudoscalar) with net Abelian charge 0 . In the semiclassical limit, both contribute to (4) as discussed in [5]. Using the GOR relation $F^{2} m_{G}^{2}=m \Sigma$ and the analytical continuation $m \rightarrow m-i \lambda / 2$, the expectation value $\mathbf{C}_{G} \equiv\left\langle\operatorname{Tr} S S^{\dagger}\right\rangle$, in (四) simplifies to

$$
\begin{gathered}
\mathbf{C}_{G}(x, y ; z, \phi) \approx+\frac{1}{2 V} \sum_{n_{\mu}} e^{i Q \cdot(x-y)} \\
\times\left(\frac{2 \Sigma}{-i \lambda+2 m+D Q^{2}}+\frac{2 \Sigma}{-i \lambda+2 m+D \tilde{Q}^{2}}\right)
\end{gathered}
$$

with the diffusion constant $D=2 F^{2} / \Sigma$, and $Q_{\mu}=n_{\mu} 2 \pi / L$ and $\tilde{Q}_{\mu}=\left(n_{\mu}+2 \phi_{\mu}\right) 2 \pi / L . \quad \Sigma=$ $|\langle\bar{q} q\rangle|$ and $F$ is the weak decay constant for the Goldstone modes of mass $m_{G}$ [7].

Since $E / \Delta=N$ and $\rho=1 / \Delta V$, with $\Sigma=\pi \rho$, we conclude after a contour integration that

$$
p(t, \phi)=\frac{1}{2} e^{-2 m|t|} \sum_{n_{\mu}}\left(e^{-D Q^{2}|t|}+e^{-D \tilde{Q}^{2}|t|}\right) .
$$

The cooperon contribution is periodic in the flux $\phi$ with periodicity $\phi=0, \pm 1 / 2, \pm 1, \ldots$. The spectrum does not discriminate between bosonic or fermionic boundary conditions in the flux-free case. The cooperon contribution $p_{C}(t, \phi)$, (second term in (7) may be rewritten using Poisson's resummation formula as

$p_{C}=\frac{V}{2(4 \pi D t)^{2}} \sum_{[l]} e^{-2 m|t|-\frac{l_{\mu}^{2} L^{2}}{4 D|t|}} \cos \left(4 \pi l_{\mu} \phi_{\mu}\right)$

with integer l's. This result is in agreement with the one derived by Montambaux [4] in disordered metals in lower dimensions. The flux-accumulation in the cooperon part implies changes in the spectral correlations of the light quarks as we now show.

\section{Spectral Rigidity}

To describe the spectral correlations associated with (11) we will use semi-classical arguments for the two-point correlation function $R(s, \phi)$ of the density of eigenvalues 3, , 4,8 . Its spectral form factor $K(t, \phi)$ is defined as

$R(s, \phi)=\int_{-\infty}^{+\infty} d t e^{i s \Delta t} K(t, \phi)$

where $\Delta=1 / \rho V$ is a typical quantum spacing at zero virtuality and in the absence of a flux. For diffusive quarks in two-color QCD, $K(t, \phi)$ relates to the return probability through [3,4,8],

$K(t, \phi) \approx \frac{\Delta^{2}|t|}{(2 \pi)^{2}} p(t, \phi)$.

For simplicity, consider the case $\phi_{\mu}=$ $(0,0,0, \phi)$ with only one-flux retained. For long proper times, the zero modes along the 1,2, 3 directions contribute only, giving

$$
\begin{gathered}
p(t, \phi)=\frac{1}{2} e^{-2 m|t|} \times \\
\sum_{n}\left(e^{-4 \pi^{2} n^{2} \sigma_{L}\left|t / t_{H}\right|}+e^{-4 \pi^{2}(n+2 \phi)^{2} \sigma_{L}\left|t / t_{H}\right|}\right)
\end{gathered}
$$

which is the analogue of a diffusion in $\mathrm{d}=1$. $\sigma_{L}=D /\left(\Delta L^{2}\right)=2(F L)^{2} / \pi$ is the dimensionless conductance, where $F^{2}$ can be regarded as the conductivity characteristic of the flow of the 
isoaxial-charge in 4+1-dimensions [3]. For $t<$ $\tau_{\text {erg }}=L^{2} / D$ the diffusive paths are short and do not accumulate enough flux. The spectral rigidity defined as [3,5] (and references therein)

$\Sigma_{2}(N, \phi)=\int_{-N}^{+N} d s(N-|s|) R(s, \phi)$

is in our case

$\Sigma_{2}(N, \phi)=\frac{1}{2 \pi^{2}} \sum_{n} \ln \left[\left(1+\frac{N^{2}}{\alpha_{n}^{2}}\right)\left(1+\frac{N^{2}}{\tilde{\alpha}_{n}^{2}}\right)\right]$

with $\alpha_{n}=\alpha+4 \pi^{2} \sigma_{L} n^{2}$ and $\tilde{\alpha}_{n}=\alpha+4 \pi^{2} \sigma_{L}(n+$ $2 \phi)^{2}$. For $N, \sigma_{L} \gg \alpha,(13)$ simplifies to

$\Sigma_{2}(N, \phi)=\Sigma_{2}(N, 0)-\frac{1}{\pi^{2}} \ln \left(1+4 \frac{\sigma_{L}}{\alpha} \sin ^{2} 2 \pi \phi\right)$

in agreement with a result derived by Montambaux [ 1 in the context of disordered metals. The conductance $\sigma_{L}$, of the chiral vacuum is directly accessible from the spectral rigidity through (14) providing for a direct measurement of this important quantity in disordered QCD. When combined with the measurement of the chiral condensate $\Sigma$, this observation allows for a novel determination of the pion weak-decay constant $F$ solely from investigations of the quark spectrum using lattice QCD.

\section{QCD Vacuum}

Since the quark return probability and the spectral rigidity are sensitive to flux-variations in a finite Euclidean volume, they could be used to probe the flux-content of the two-color QCD vacuum, provided that the fluxes upset the pseudoreal character of the quark fields. Indeed, if we consider an Abelian flux-disordered vacuum along the lines we have so far discussed, and characterized by a Gaussian distributed flux with a mean

$$
\left\langle\left\langle\phi_{\mu} \phi_{\nu}\right\rangle\right\rangle=\kappa_{\mu}^{2} \delta_{\mu \nu}
$$

then the quark return probability can be easily estimated from (7) using the Poisson form (8) for the cooperon part. If we split the quark return probability $p=p_{D}+p_{C}$, then the diffuson part $p_{D}$, is flux-insensitive

$$
\left\langle\left\langle p_{D}(t, \phi)\right\rangle\right\rangle=\frac{V}{2(4 \pi D t)^{2}} e^{-2 m|t|}
$$

while the cooperon part $p_{C}(t, \phi)$, is flux-sensitive

$$
\left\langle\left\langle p_{C}\right\rangle\right\rangle=\frac{V}{2(4 \pi D t)^{2}} \sum_{[l]} e^{-2 m|t|-\frac{l_{\mu}^{2} L^{2}}{4 D|t|}-8 \pi^{2} \kappa_{\mu}^{2} l_{\mu}^{2}} .
$$

We note that the periodicity in $\phi=0, \pm 1 / 2, \pm 1, \ldots$ of the quark return probability implies that the latter is likely to be insensitive to a $Z_{2}$ fluxdisordered vacuum. If these effects extend to an Abelian flux-rich vacuum, a simple way to detect them is to measure the relative ratio of the quark return probabilities for $N_{c}=2$ and $N_{c}=3$. A flux sensitivity implies a t-dependent ratio close to $1 / 2$ (as opposed to 1 ), assuming that the vacuum structure does not change appreciably from two to three colors.

\section{Acknowledgments}

This work was supported in part by the US DOE grants DE-FG-88ER40388 and DE-FG0286ER40251, by the Polish Government Project (KBN) grants 2P03B01917 and 2P03B00814 and by the Hungarian grant OTKA-T022931.

\section{REFERENCES}

1. M. Peskin, Nucl. Phys. B175 (1980) 197, and references therein.

2. H. Leutwyler and A. Smilga, Phys. Rev. D46 (1992) 5607.

3. R.A. Janik, M.A. Nowak, G. Papp and I. Zahed, Phys. Rev. Lett. 81 (1998) 264.

4. G. Montambaux, cond-mat/9602071, and references therein.

5. R.A. Janik, M.A. Nowak, G. Papp and I. Zahed, hep-ph/9807499

6. Throughout we assume that the light baryonantibaryon fluctuations do not dynamically 'screen' the Abelian fluxes. This is evident at $\phi=1 / 2$ where there is only a net change in the statistics. This assumption is not needed in the quenched case.

7. The implicit flux dependence of the present parameters at next to leading order is subsumed.

8. N. Argaman, Y. Imry and U. Smilansky, Phys. Rev. B47 (1993) 4440. 